\title{
Millimeter Wave Communications over Relay Networks
}

DOI:

10.1109/WCNC.2017.7925836

\section{Document Version}

Accepted author manuscript

Link to publication record in Manchester Research Explorer

\section{Citation for published version (APA):}

Abbas, H., \& Hamdi, K. (2017). Millimeter Wave Communications over Relay Networks. In 2017 IEEE Wireless Communications and Networking Conference https://doi.org/10.1109/WCNC.2017.7925836

\section{Published in:}

2017 IEEE Wireless Communications and Networking Conference

\section{Citing this paper}

Please note that where the full-text provided on Manchester Research Explorer is the Author Accepted Manuscript or Proof version this may differ from the final Published version. If citing, it is advised that you check and use the publisher's definitive version.

\section{General rights}

Copyright and moral rights for the publications made accessible in the Research Explorer are retained by the authors and/or other copyright owners and it is a condition of accessing publications that users recognise and abide by the legal requirements associated with these rights.

\section{Takedown policy}

If you believe that this document breaches copyright please refer to the University of Manchester's Takedown Procedures [http://man.ac.uk/04Y6Bo] or contact uml.scholarlycommunications@manchester.ac.uk providing relevant details, so we can investigate your claim.

\section{OPEN ACCESS}




\title{
Millimeter Wave Communications over Relay Networks
}

\author{
Hatem Abbas, and Khairi Hamdi, \\ School of Electrical and Electronic Engineering, \\ The University of Manchester, Manchester, UK. \\ emails: hatem.h.abbas@ieee.org, k.hamdi@manchester.ac.uk
}

\begin{abstract}
Relays are expected be an important factor in the implementation of millimeter wave (mmWave) based cellular networks due to the limited coverage of mmWave communications. Adding a single or multiple relays in the link between two nodes is an inevitable step towards high capacity wireless access and/or backhaul links. In this paper we suggest employing two-hop multiple relay networks as a solution to link two points using mmWave frequencies. Hybrid (Analog and Digital) precoding is used at the two terminal nodes with analog beamformers/combiners at the relays. The proposed design algorithm requires no channel information with relatively limited training overhead. The performance of the system is examined under different channel and system layouts. The results highlight the positive impact of employing relays in mmWave link as well as the effect of array size on the overall system performance.

Index Terms-Millimeter wave, Hybrid beamforming, Relay
\end{abstract} Networks, Channel Sounding, 5G.

\section{INTRODUCTION}

In a few years time, cellular networks will be required to provide 1000 times the throughput provided today [1]. In order to meet such high demands, three scenarios are suggested: increasing the frequency bandwidth, enhancing the spectral efficiency, and more geographical re-usability [2], [3]. The relatively wide bandwidth and the introduction of the cost efficient CMOS technology make mmWave a perfect fit to be the core of the next generation communication.

Despite its many advantages, $60 \mathrm{GHz}$ frequencies suffer from relatively high propagation losses. Several researchers investigated ways to overcome these losses [4]-[6]. One of the proposed solutions is to increase the directivity gain of the systems using analog, digital, or hybrid (analog and digital) beamformers [7]. Others include changing the layout of the cellular system by introducing dense networks, and using cooperative networks [8], [9].

The complexity of the digital beamformers (DBF) and the limitations of the analog beamformers $(\mathrm{ABF})$ make hybrid beamformers $(\mathrm{HBF})$ an interesting solution to provide the required directivity. The number of the RF chains in hybrid systems is less than the number of antennas which makes hybrid systems cost efficient [10]. One of the popular methods to jointly design the analog and digital processors in $\mathrm{HBF}$ systems was introduced by [14]. The proposed design algorithm uses the sparsity of the mmWave channel and orthogonal match pursuit (OMP) [13] principle while assuming full CSI. This method gained importance because of the low complexity of the design [15].

Furthermore, relay networks attract a great deal of research interest in recent years as an architecture to increase the system performance [16]. The study of two-hop multiple single-antenna relay networks was introduced in [17], and the optimization of the MMSE solution for MIMO relay networks has been investigated in [18]. In millimeter waves, there is a shortage of the research in single relay networks, rather than multiple relays. In a recent study, half duplex (HD) relay network was introduced in mmWave environment where the sparsity principle is used to jointly design the hybrid beamforming processors at the relay [19]. However, multiple relays network have not been studied throughly.

Combining the benefits of the wide mmWave spectrum with the flexibility provided by the multiple relay networks is a key solution in designing small dense cells architecture that secures the high throughput needed. In this paper, we study the performance of hybrid beamformers in the two-hop multiple relay networks in mmWave. A split domain algorithm is proposed inspired by the performance of the well known sparsity based algorithm for HBF design [14], [20] and the channel sounding techniques for ABF [21], [22].

The main contribution of this work is the study of multi-relay networks in mmWave and developing the beamformers design algorithm. The work will be extended to include comparing different search techniques providing power and time saving. The rest of the paper organized as follows. The system and channel models with the main assumptions used in the paper are presented in Section II. The design of the hybrid and analog beamformers is presented in Section III. Simulation results are presented in Section IV before concluding the paper in Section V.

We use the following notation throughout this paper: $\mathbf{A}$ is a matrix; $\mathbf{a}$ is a vector; $a$ is a scalar. $\mathbf{A}^{(i)}$ is the $i^{t h}$ column of $\mathbf{A}, \mathbf{A}_{(i, j)}$ is the $(i, j)$-th entry of $\mathbf{A}$. $\|\mathbf{A}\|_{F}$ is the Frobenius norm of $\mathbf{A}$, whereas $(\mathbf{A})^{T},(\mathbf{A})^{H},(\mathbf{A})^{-1}$ denote transpose, Hermitian, and inverse respectively. $\|\mathbf{a}\|_{p}$ is the $p$-norm of a. $\mathbf{I}_{N}$ is the $N \times N$ identity matrix; $\mathbf{0}_{M \times N}$ is the $M \times N$ all-zeros matrix. $\mathcal{C N}(\mathrm{a} ; \mathbf{A})$ is a complex Gaussian vector with mean a and covariance matrix $\mathbf{A}$. 


\section{SyStem AND ChANNEL Models}

\section{A. Network Model}

Consider a multi relay network consists of one source, one destination, and $K$ relays, as illustrated in Fig. 1. The source \{destination $\}$ is equipped with $N_{\mathrm{T}}\left\{N_{\mathrm{R}}\right\}$ antennas and $M_{\mathrm{T}}$ $\left\{M_{\mathrm{R}}\right\}$ RF chains. Each relay has $N_{\mathrm{R}}^{r} / N_{\mathrm{T}}^{r}$ receive/transmit antennas and a single RF chain. In this paper, we consider source-destination connection via one stream through each relay (i.e. $N_{S}=K$ ). We assume limited number of relays where $K \leq \min \left\{M_{\mathrm{R}}, M_{\mathrm{T}}\right\}$. It is also assumed the source uses only $K$ out of the available RF chains.

\section{B. Beamforming System Setup}

Using the available multiple RF chains, both the source and the destination process the signal in the analog and digital domains. During the transmission, the source applies the $N_{S} \times$ $M_{\mathrm{T}}$ digital precoder $\mathbf{F}_{\mathrm{D}}=\left[\mathbf{f}_{1}^{\mathrm{D}}, \mathbf{f}_{2}^{\mathrm{D}}, \cdots, \mathbf{f}_{M_{\mathrm{T}}}^{\mathrm{D}}\right]$ followed by an $N_{\mathrm{T}} \times M_{\mathrm{T}}$ analog precoder $\mathbf{F}_{\mathrm{A}}=\left[\mathbf{f}_{1}^{\mathrm{A}}, \mathbf{f}_{2}^{\mathrm{A}}, \cdots, \mathbf{f}_{M_{\mathrm{T}}}^{\mathrm{A}}\right]$ on the transmit data. Therefore, the $k$-th relay received signal will be

$$
\mathbf{y}_{r, k}=\mathbf{H}_{k} \sum_{i=1}^{K} \mathbf{F}_{\mathrm{A}} \mathbf{f}_{i}^{\mathrm{D}} s_{i}+\mathbf{n}_{1, k}
$$

where $\mathbf{s}=\left[s_{1} \cdots s_{K}\right]$ is the $N_{S} \times 1$ transmit signal with $\mathbb{E}\left[\mathbf{s s}^{H}\right]=\frac{\rho}{N_{S}} \mathbf{I}_{N_{S}}$, where $\rho$ is the total transmitted power. $\mathbf{H}_{k}$ is the mmWave MIMO channel between the source and the $k$ th relay, $\mathbf{n}_{1, k}$ is the additive noise vector with covariance matrix $\mathcal{R}_{n_{1, k}}=\sigma_{n_{1, k}}^{2} \mathbf{I}_{N_{\mathrm{R}}^{r}}$.

Each relay has an analog combiner $\mathbf{c}_{k}$ to receive the signal, and an analog beamformer $\mathbf{b}_{k}$ for transmission. The $k$ th relay transmitted signal can be described as:

$$
\mathbf{x}_{r, k}=\mathbf{b}_{k} \mathbf{c}_{k}^{H} \mathbf{y}_{r, k}
$$

the destination observes the signals transmitted by the relays and the received signal can be written as

$$
\begin{aligned}
\mathbf{y}=\sum_{k=1}^{K}\left(\mathbf{G}_{k} \mathbf{b}_{k} \mathbf{c}_{k}^{H}\right. & \mathbf{H}_{k} \sum_{i=1}^{K} \mathbf{F}_{\mathrm{A}} \mathbf{f}_{i}^{\mathrm{D}} s_{i} \\
& \left.+\mathbf{G}_{k} \mathbf{b}_{k} \mathbf{c}_{k}^{H} \mathbf{n}_{1, k}\right)+\mathbf{n}_{2}
\end{aligned}
$$

where $\mathbf{G}_{k}$ is the mmWave channel between the $k$ th relay and the destination, and $\mathbf{n}_{2}$ is the additive white noise at the destination with covariance matrix $\mathcal{R}_{n_{2}}=\sigma_{n_{2}}^{2} \mathbf{I}_{N_{\mathrm{R}}}$. Finally, the destination uses hybrid beamforming to process the received signal. The estimated signal can be written as

$$
\begin{aligned}
\hat{\mathbf{s}}= & \mathbf{W}^{H} \mathbf{G B C}^{H} \mathbf{H} \mathbf{F}_{\mathrm{A}} \mathbf{F}_{\mathrm{D}} \mathbf{s}+\mathbf{W}^{H} \mathbf{G B C}^{H} \mathbf{n}_{1} \\
& +\mathbf{W}^{H} \mathbf{n}_{2}
\end{aligned}
$$

where $\mathbf{W}=\mathbf{W}_{\mathrm{A}} \mathbf{W}_{\mathrm{D}}$, with $\mathbf{W}_{\mathrm{A}}$ being the analog combiner, and $\mathbf{W}_{\mathrm{D}}$ is the digital processor. $\mathbf{H}=\left[\mathbf{H}_{1}^{T}, \mathbf{H}_{2}^{T}, \cdots, \mathbf{H}_{K}^{T}\right]^{T}$ is the $K N_{\mathrm{R}}^{r} \times N_{\mathrm{T}}$ channel matrix between the source and the relays, $\mathbf{n}_{1}=\left[\mathbf{n}_{1,1}^{T}, \mathbf{n}_{1,2}^{T}, \cdots, \mathbf{n}_{1, K}^{T}\right]^{T}$ is the $K N_{\mathrm{R}}^{r} \times 1$ noise matrix at the relays, $\mathbf{G}=\left[\mathbf{G}_{1}, \mathbf{G}_{2}, \cdots, \mathbf{G}_{K}\right]$ is the $N_{\mathrm{R}} \times$ $K N_{\mathrm{T}}^{r}$ channel matrix between the all the relay nodes and the

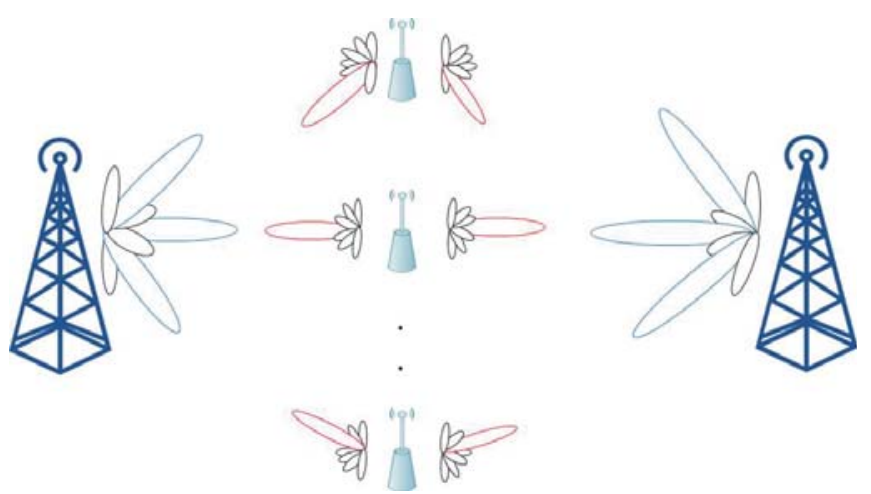

Fig. 1: Multi-relay system with hybrid and analog beamforming

destination, $\mathbf{C}=\left[\mathbf{c}_{1}^{T}, \mathbf{c}_{2}^{T}, \cdots, \mathbf{c}_{K}^{T}\right]^{T}$ is the $K N_{\mathrm{R}}^{r} \times 1$ equivalent all relays combiner matrix, and $\mathbf{B}=\left[\mathbf{b}_{1}^{T}, \mathbf{b}_{2}^{T}, \cdots, \mathbf{b}_{K}^{T}\right]^{T}$ is the $K N_{\mathrm{T}}^{r} \times 1$ equivalent all relays beamformer matrix.

The analog beamformers are implemented using analog phase shifters, therefore, their entries have equal norm, i.e. at the source $\left(\mathbf{F}_{\mathrm{A}}^{(i)} \mathbf{F}_{\mathrm{A}}^{(i) H}\right)_{(\ell, \ell)}=N_{\mathrm{T}}^{-1}$. On the other hand, digital beamformer at the source, $\mathbf{F}_{\mathrm{D}}$, has no hardware constraints except the total power constraint. With the limitation on the analog beamformers hardware, such as choosing the phase shifters angles out of quantized sets, the analog beamformer vectors need to be chosen from finite size codebooks. The codebook size will effect the duration of the channel training as will be explained shortly in Section III.

\section{Channel Model}

The measurements of outdoor mmWave $28 \mathrm{GHz}$ and the $72 \mathrm{GHz}$ channels showed that they normally have limited scattering [23], [24] where the multipath components are mainly generated by reflection. To incorporate this effect, we adopt a geometric channel model based on the extended SalehValenzuela model in which the channel is assumed to be a sum of $L$ scattering clusters, each of which contributes $P$ propagation paths to the channel [25], [26]. With half-wave spaced uniform linear arrays (ULAs) used at the transmitter and the receiver, the channel matrix can be expressed as

$$
\mathbf{H}_{k}\left\{\mathbf{G}_{k}\right\}=\frac{1}{\sqrt{P L}} \sum_{\ell=1}^{L} \sum_{p=1}^{P} \alpha_{p \ell} \mathbf{a}_{r}\left(\theta_{r}^{p \ell}\right) \mathbf{a}_{t}^{*}\left(\theta_{t}^{p \ell}\right),
$$

where $\alpha_{p \ell}$ is the complex small-scale fading gain of the $p$ th sub-path in the $\ell$-th cluster, $\theta_{p \ell}^{r}$ and $\theta_{p \ell}^{t} \in\{0,2 \pi\}$ are the angles of arrival and departure (AoA/AoD), respectively. The gains $\alpha_{p \ell}$ are complex Gaussian random variables with zero mean and variance $\sigma_{\alpha}^{2}$. The mean angle associated with each cluster is uniformly distributed over $[-\pi, \pi]$, and the distribution of the difference between an AoA $\{$ AoD $\}$ and its mean is Laplacian with angular standard deviation $\gamma$. The vectors $\mathbf{a}_{r}\left(\theta_{p \ell}^{r}\right)$ and $\mathbf{a}_{t}^{H}\left(\theta_{p \ell}^{t}\right)$ are the array response vectors at the receiver and transmitter, respectively.

Without loss of generality, we are using ULA to get the results 
in the simulation in Section IV. In this case, the array response vector (a) is defined as

$$
\mathbf{a}(\theta)=\frac{1}{\sqrt{M}}\left[\begin{array}{llll}
1 & e^{j 2 \pi \frac{d}{\lambda} \sin (\theta)} \cdots e^{j 2 \pi(M-1) \frac{d}{\lambda} \sin (\theta)}
\end{array}\right]^{T}
$$

where $d$ is the space between the antennas, $M$ is the number of antennas, and $\lambda$ is the wavelength.

\section{BEAMFORMERS DESIGN}

This work aims to effectively design the hybrid beamformer at the source and the destination as well as the analog beamformers at the relays in order to maximize the achievable rate at the destination. Assuming Gaussian transmission, the achievable rate could be written as described in (7), at the top of the next page.

Maximizing the rate requires joint design of the $(2 K+4)$ beamforming matrices. However, the constraints on the analog beamformers make finding the joint solution untraceable [14], [15]. In this work, we propose splitting the design into two domains: analog and digital. The design will be performed over two phases, the first phase involves designing the analog beamforming matrices. Then, during the second phase, the digital processors are designed.

\section{A. Phase One: Analog Domain}

The analog beamforming matrices are designed by choosing the best beamforming vectors from sets of predefined vectors known as codebooks. The process includes repeatedly transmitting training signal and search for the vector pair that maximizes a measuring factor (we use beamforming gain as the measuring factor in this work). This process is known as channel sounding (CS) and it is performed without the need for CSI using relatively low overhead [15], [21]. Exhaustive search (ES) where the all the possible beamforming/combining vectors combinations are tested is used to search for the best pair. ES is considered the most straight forward search technique, yet it has higher overhead as compared to other search techniques. Optimistically, if the analog beamformers have continuous angles capabilities and the infinite codebook size, the selected vectors will have the same angles as the AoA/AoD. Comparing different adaptive and non-adaptive search techniques to provide time and power saving is a natural extension to this work.

Each node of the system has its own codebook to use during the channel sounding. $\mathcal{B}, \mathcal{C}$ represent the transmitting and receiving codebooks at the relay, respectively. $\mathcal{F}, \mathcal{W}$ represent source, destination codebooks. We also propose using sectored codebooks where the source and destination sectorize the available spectrum over the available relays, assuming the relays are distributed uniformly and equally distanced. $\mathcal{F}_{k}$ and $\mathcal{W}_{k}$ represent the sectored codebook assigned to the $k$ th relay at the source and destination, respectively. The duration of the sounding period depends on the size of the codebook, for instance, the duration of the sounding between the source and the $k$ th relay will be $T=N_{\mathcal{F}_{k}} \times N_{\mathcal{C}}$, where $N_{a}$ is the number of columns in $a$, or codebook size.
At each iteration of phase one, a relay combiner $\mathbf{c}_{k}$, beamformer $\mathbf{b}_{k}$, and the related source \{destination\} beamformer $\{$ combiner $\} \mathbf{f}_{k}^{\mathrm{A}}\left\{\mathbf{w}_{k}^{\mathrm{A}}\right\}$ are found. After $K$ iterations, $\mathbf{F}_{\mathrm{A}}$, $\mathbf{W}_{\mathrm{A}}, \mathbf{b}_{i}$, and $\mathbf{c}_{i}(1 \leq i \leq K)$ are all constructed. The first phase is concluded by calculating the effective channels $\tilde{\mathbf{h}}_{k}=\mathbf{c}_{k}^{H} \mathbf{H}_{k} \mathbf{F}_{\mathrm{A}}$, and $\tilde{\mathbf{g}}_{k}=\mathbf{W}_{\mathrm{A}}^{H} \mathbf{G}_{k} \mathbf{b}_{k}(1 \leq k \leq K)$ to be used in the digital processor design.

\section{B. Phase Two: Digital Domain}

During the second phase, the source and destination design their zero-forcing (ZF) digital processor using the effective channel calculated in the previous stage. Due to the sparse nature of the mmWave channel and the sectored beamforming, the effective channels are expected to be well-conditioned to be used in designing the digital processors using $\mathrm{ZF}$ technique. The design procedure is outlined in Algorithm 1. The design is concluded by satisfying the power condition for the digital processor at the source.

\section{Simulation Results}

The performance of the proposed algorithm is examined by measuring the achievable rate through computer simulations. All the relays are assumed to have the same distance from the source and destination so that all the channels have the same statistics. Each of the propagation channels is modeled with $L$ clusters channel and each cluster contributes with single ray with uniformly distributed AoAs and AoDs. The complex path gains are assumed to be Gaussian distributed with equal variances. We assume a ULA with inter-elements spacing $d=\lambda / 2$. The noise variance $\sigma_{n_{1, k}}^{2}=\sigma_{n_{2}}^{2}$, and the signal to noise ratio is defined as $S N R=\frac{\rho}{N_{S} \sigma_{n}^{2}}$. The coverage of each node is specified with $\theta \in\left\{-\frac{\pi}{2}, \frac{\pi}{2}\right\}$.

Fig. 2 shows the performance of the system under single path channel as compared to the optimistic results where the CS uses continuous angles. Two different size codebooks are used, one with 7 vectors and the other with 11 vectors. Adding vectors to the codebook improve the performance and eventually the system performance will approach the optimistic results. However, this comes on the price of the time as the sounding duration for the larger codebook is 3 times the duration of the other. ULAs with 16 antennas are used at the source, destination, and each of the relays.

The effect of the number of the relays is examined in Fig. 3. The results are obtained using channels with $L=20,40$ paths. Having more relays in the network increases the probability of reaching the destination, thus higher spectral efficiency. Fig. 4 compares the system performance for different array size at source/destination with fixed relay arrays at 8 antennas on each side. Increasing the number of antennas in the array will improve the directivity, which leads to better performance. However, the capacity to improve the performance is limited by the size of the relays arrays.

Finally, the performance of the proposed algorithm is examined using channels with more than single path as shown in Fig. 5. The results indicate a similar behavior to that with 


$$
R=\log _{2}\left(1+\frac{\rho\left|\mathbf{W}_{\mathrm{D}}^{H} \mathbf{W}_{\mathrm{A}}^{H} \sum_{k=1}^{K} \mathbf{G}_{k} \mathbf{b}_{k} \mathbf{c}_{k}^{H} \mathbf{H}_{k} \mathbf{F}_{\mathrm{A}} \mathbf{f}_{k}^{\mathrm{D}}\right|^{2}}{\rho\left|\mathbf{W}_{\mathrm{D}}^{H} \mathbf{W}_{\mathrm{A}}^{H} \sum_{k=1}^{K} \sum_{j \neq k}^{K} \mathbf{G}_{k} \mathbf{b}_{k} \mathbf{c}_{k}^{H} \mathbf{H}_{k} \mathbf{F}_{\mathrm{A}} \mathbf{f}_{j}^{\mathrm{D}}\right|^{2}+\left|\mathbf{W}_{\mathrm{D}}^{H} \mathbf{W}_{\mathrm{A}}^{H}\right|^{2}+1}\right)
$$

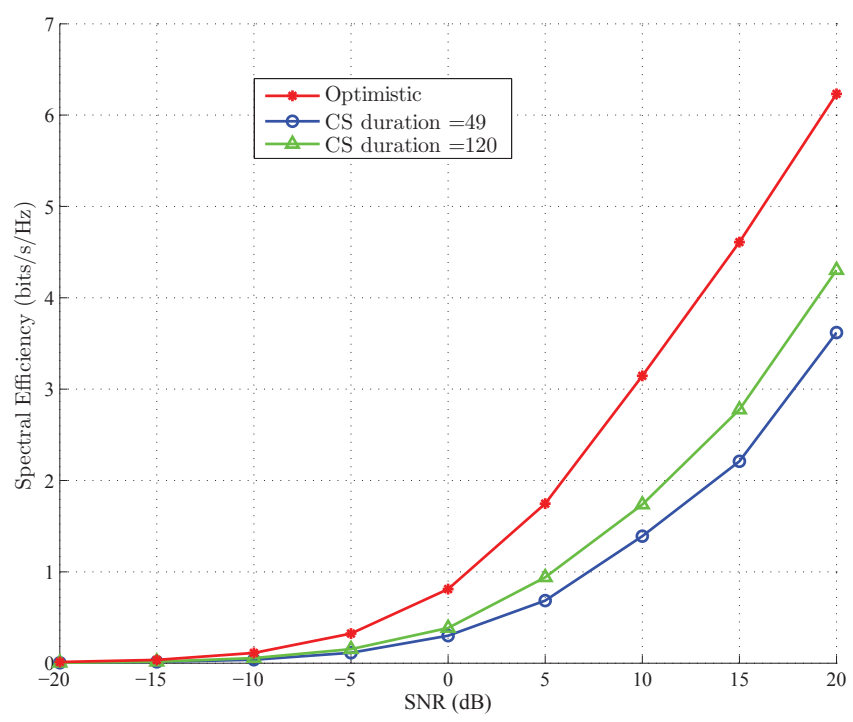

Fig. 2: Spectral efficiency versus SNR with $L=1, K=4$ relays, and each node of the network has 16 antennas.

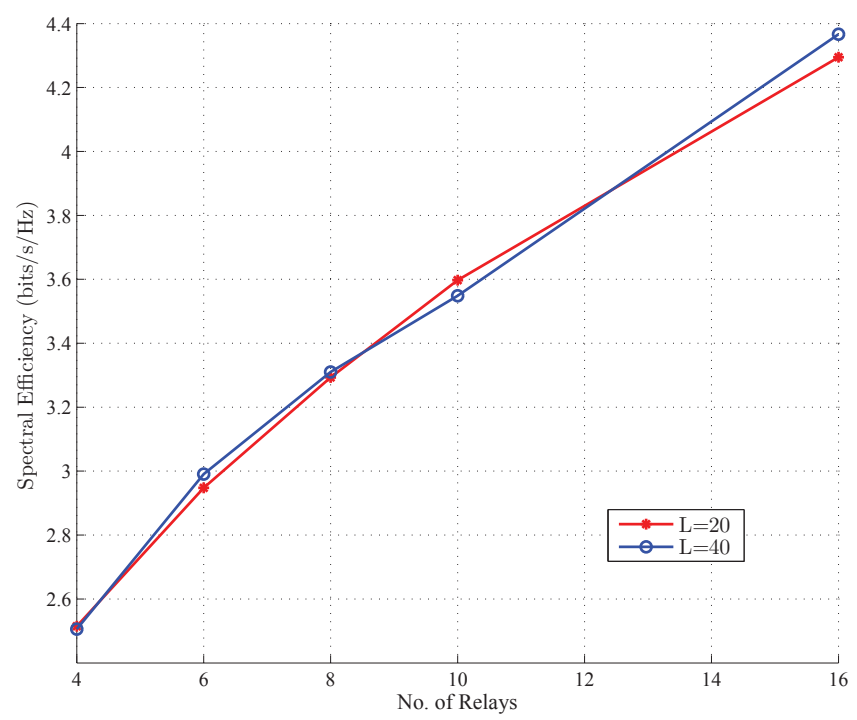

Fig. 3: Spectral efficiency versus number of relays when $L \in$ $\{20,40\}$, and $\mathrm{SNR}=5 \mathrm{~dB}$.
Algorithm 1 Analog and digital beamformers design by splitting into analog and digital design phases

\section{Input: analog sets:}

$\mathcal{F}=\left[\mathbf{f}_{1}, \mathbf{f}_{2}, \cdots, \mathbf{f}_{N_{\mathcal{F}}}\right], \mathcal{W}=\left[\mathbf{w}_{1}, \mathbf{w}_{2}, \cdots, \mathbf{w}_{N_{\mathcal{W}}}\right]$,

$\mathcal{C}=\left[\mathbf{u}_{1}, \mathbf{u}_{2}, \cdots, \mathbf{u}_{N_{\mathcal{C}}}\right], \mathcal{B}=\left[\mathbf{v}_{1}, \mathbf{v}_{2}, \cdots, \mathbf{v}_{N_{\mathcal{B}}}\right]$

Output: $\mathbf{F}_{\mathrm{A}}, \mathbf{W}_{\mathrm{A}}, \mathbf{b}_{i}, \mathbf{c}_{i}(1 \leq i \leq K)$

Initializing: Sectorize $\mathcal{F}$ and $\mathcal{W}$ based on the number of relays

- Phase One: Design the analog processors.

- Set the analog sets for each relay

- Search for the optimum pair that maximize the gain: * source-relay

$$
\left(\mathbf{f}_{k}^{\mathrm{A}}, \mathbf{c}_{k}\right)=\underset{\substack{\forall \mathbf{f} \in \mathcal{F}_{k} \\ \forall \mathbf{u} \in \mathcal{C}}}{\operatorname{argmax}}\left|\mathbf{u}^{H} \mathbf{H}_{k} \mathbf{f}\right|^{2}
$$

* relay-destination

$$
\left(\mathbf{w}_{k}^{\mathrm{A}}, \mathbf{b}_{k}\right)=\underset{\substack{\forall \mathbf{w} \in \mathcal{W}_{k} \\ \forall \mathbf{v} \in \mathcal{B}}}{\operatorname{argmax}}\left|\mathbf{w}^{H} \mathbf{H}_{k} \mathbf{v}\right|^{2}
$$

- Compute the effective channels at both sides as $\tilde{\mathbf{h}}_{k}=$ $\mathbf{c}_{k}^{H} \mathbf{H}_{k} \mathbf{F}_{\mathrm{A}}$, and $\tilde{\mathbf{g}}_{k}=\mathbf{W}_{\mathrm{A}}^{H} \mathbf{G}_{k} \mathbf{b}_{k}$

- Phase Two: Design the digital processor at the source and destination.

- Define the effective first hop and second hop channels as $\tilde{\mathbf{H}}=\left[\tilde{\mathbf{h}}_{1}^{T}, \tilde{\mathbf{h}}_{2}^{T}, \cdots, \tilde{\mathbf{h}}_{K}^{T}\right]^{T}$ and $\tilde{\mathbf{G}}=$ $\left[\tilde{\mathbf{g}}_{1}, \tilde{\mathbf{g}}_{2}, \cdots, \tilde{\mathbf{g}}_{K}\right]$, respectively.

- Design $\mathbf{F}_{\mathrm{D}}$ and $\mathbf{W}_{\mathrm{D}}$ using $\mathrm{ZF}$ :

$$
\begin{aligned}
\mathbf{F}_{\mathrm{D}} & =\tilde{\mathbf{H}}^{H}\left(\tilde{\mathbf{H}} \tilde{\mathbf{H}}^{H}\right)^{-1} \\
\mathbf{W}_{\mathrm{D}} & =\tilde{\mathbf{G}}^{H}\left(\tilde{\mathbf{G}} \tilde{\mathbf{G}}^{H}\right)^{-1}
\end{aligned}
$$

- Compute the final digital processors that satisfy the power constraints.

$$
\mathbf{f}_{k}^{\mathrm{D}}=\frac{\mathbf{f}_{k}^{\mathrm{D}}}{\left\|\mathbf{F}_{\mathrm{A}} \mathbf{f}_{k}^{\mathrm{D}}\right\|_{F}} \quad \forall k
$$

single path channel. Adding more relays have a positive impact on the performance.

\section{CONCLUSION}

In this paper, we propose a two phase split algorithm for two-hop multiple relays networks in millimeter waves. The source and the destination are equipped with hybrid beamformers and multiple RF chains, while each of the relays is equipped with single RF chain and an analog beamformer. 


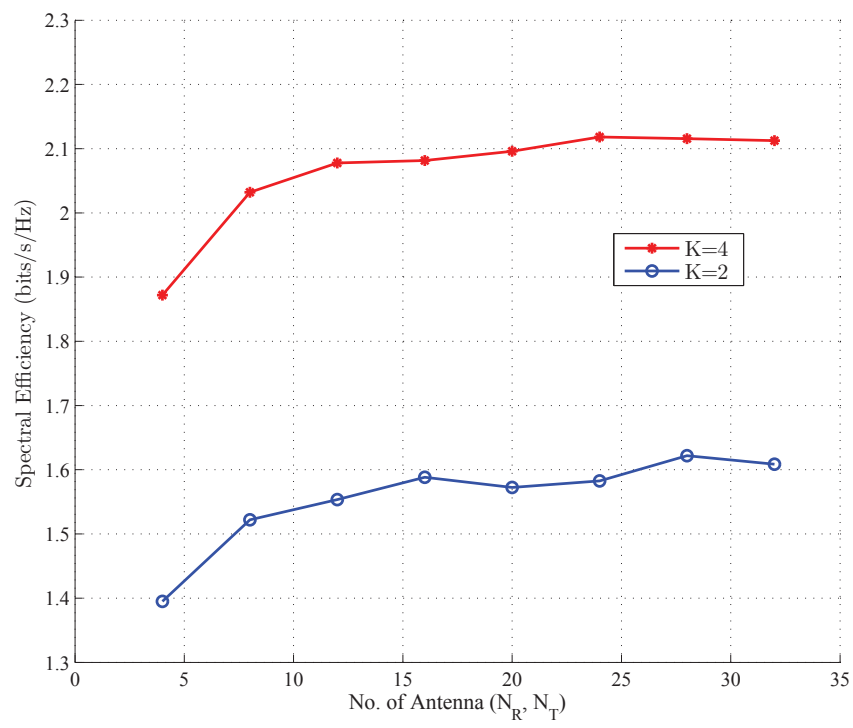

Fig. 4: Spectral efficiency versus array size at the source and destination. Number of antennas at each relay fixed to $N_{\mathrm{T}}^{r}=$ $N_{\mathrm{R}}^{r}=8 . K \in\{2,4\}, \mathrm{SNR}=5 \mathrm{~dB}$, and $L=10$.

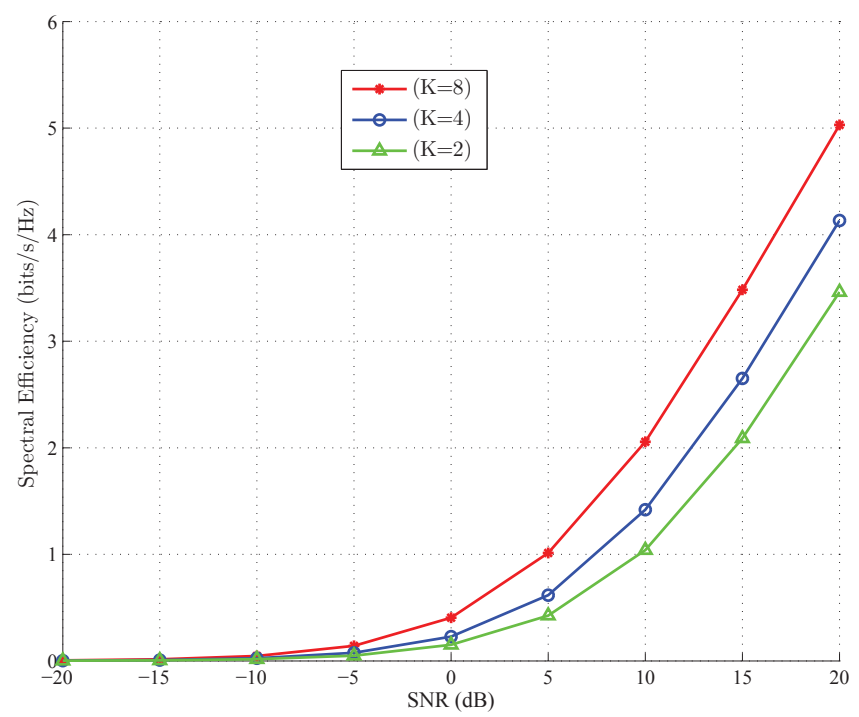

Fig. 5: Spectral efficiency versus training duration for the analog beamforming. array size $N_{\mathrm{T}}=N_{\mathrm{R}}=16, N_{\mathrm{T}}^{r}=N_{\mathrm{R}}^{r}=8$. $K \in\{2,4\}$, and $L=10$.

The beamformers work on directing the signal to obtain higher beamforming gains help overcoming the path losses in outdoors mmWave environments. The performance of the system is compared to the optimistic results where the analog beamformers have continuous angles capabilities. The analog beamformers/combiners are designed using the modified joint channel sounding by sectoring the coverage spectrum based on the number of relays.

The results showed that the array size, the codebook size and the number of the relays in the system have positive impact on the system performance.

\section{REFERENCES}

[1] S. Rangan, T. Rappaport, and E. Erkip, "Millimeter-wave cellular wireless networks: Potentials and challenges," Proceedings of the IEEE, vol. 102, no. 3, pp. 366-385, March 2014.

[2] C. Jeong, J. Park, and H. Yu, "Random access in millimeter-wave beamforming cellular networks: issues and approaches," Communications Magazine, IEEE, vol. 53, no. 1, pp. 180-185, January 2015.

[3] C. Dehos, J. Gonzalez, A. De Domenico, D. Ktenas, and L. Dussopt "Millimeter-wave access and backhauling: the solution to the exponential data traffic increase in $5 \mathrm{~g}$ mobile communications systems?" Communications Magazine, IEEE, vol. 52, no. 9, pp. 88-95, September 2014.

[4] D. Lockie and D. Peck, "High-data-rate millimeter-wave radios," $\mathrm{Mi}$ crowave Magazine, IEEE, vol. 10, no. 5, pp. 75-83, August 2009.

[5] A. Sulyman, A. Nassar, M. Samimi, G. MacCartney, T. Rappaport, and A. Alsanie, "Radio propagation path loss models for $5 \mathrm{~g}$ cellular networks in the $28 \mathrm{ghz}$ and $38 \mathrm{ghz}$ millimeter-wave bands," Communications Magazine, IEEE, vol. 52, no. 9, pp. 78-86, September 2014.

[6] P. Wang, Y. Li, L. Song, and B. Vucetic, "Multi-gigabit millimeter wave wireless communications for 5G: from fixed access to cellular networks," Communications Magazine, IEEE, vol. 53, no. 1, pp. 168-178, January 2015.

[7] W. Roh, J.-Y. Seol, J. Park, B. Lee, J. Lee, Y. Kim, J. Cho, K. Cheun, and F. Aryanfar, "Millimeter-wave beamforming as an enabling technology for $5 \mathrm{~g}$ cellular communications: theoretical feasibility and prototype results," Communications Magazine, IEEE, vol. 52, no. 2, pp. 106-113, February 2014

[8] R. Baldemair, T. Irnich, K. Balachandran, E. Dahlman, G. Mildh Y. Selen, S. Parkvall, M. Meyer, and A. Osseiran, "Ultra-dense networks in millimeter-wave frequencies," Communications Magazine, IEEE, vol. 53, no. 1, pp. 202-208, January 2015.

[9] U. Siddique, H. Tabassum, E. Hossain, and D. I. Kim, "Wireless backhauling of $5 \mathrm{~g}$ small cells: challenges and solution approaches," Wireless Communications, IEEE, vol. 22, no. 5, pp. 22-31, October 2015.

[10] S. Han, C.-L. I, Z. Xu, and C. Rowell, "Large-scale antenna systems with hybrid analog and digital beamforming for millimeter wave $5 \mathrm{~g}$," Communications Magazine, IEEE, vol. 53, no. 1, pp. 186-194, January 2015.

[11] V. Venkateswaran and A.-J. van der Veen, "Analog beamforming in mimo communications with phase shift networks and online channel estimation," Signal Processing, IEEE Transactions on, vol. 58, no. 8, pp. 4131-4143, Aug 2010

[12] J. Nsenga, A. Bourdoux, W. Van Thillo, V. Ramon, and F. Horlin, "Joint tx/rx analog linear transformation for maximizing the capacity at 60 ghz," in Communications (ICC), 2011 IEEE International Conference on, June 2011, pp. 1-5.

[13] J. Tropp and S. Wright, "Computational methods for sparse solution of linear inverse problems," Proceedings of the IEEE, vol. 98, no. 6, pp. 948-958, June 2010.

[14] O. El Ayach, S. Rajagopal, S. Abu-Surra, Z. Pi, and R. Heath, "Spatially sparse precoding in millimeter wave mimo systems," Wireless Coтmunications, IEEE Transactions on, vol. 13, no. 3, pp. 1499-1513, March 2014.

[15] A. Alkhateeb, G. Leus, and R. Heath, "Limited feedback hybrid precoding for multi-user millimeter wave systems," Wireless Communications, IEEE Transactions on, vol. PP, no. 99, 2015.

[16] A. Toding, M. R. Khandaker, and Y. Rong, "Joint source and relay optimization for parallel mimo relay networks," EURASIP Journal on Advances in Signal Processing, vol. 2012, no. 1, pp. 1-7, 2012.

[17] N. Khajehnouri and A. H. Sayed, "Distributed mmse relay strategies for wireless sensor networks," IEEE Transactions on Signal Processing, vol. 55, no. 7, pp. 3336-3348, July 2007.

[18] A. S. Behbahani, R. Merched, and A. M. Eltawil, "Optimizations of a mimo relay network," IEEE Transactions on Signal Processing, vol. 56, no. 10 , pp. 5062-5073, Oct 2008.

[19] J. Lee and Y. Lee, "Af relaying for millimeter wave communication systems with hybrid rf/baseband mimo processing," in Communications (ICC), 2014 IEEE International Conference on, June 2014, pp. 58385842 
[20] A. Alkhateeb, O. El Ayach, G. Leus, and R. Heath, "Channel estimation and hybrid precoding for millimeter wave cellular systems," Selected Topics in Signal Processing, IEEE Journal of, vol. 8, no. 5, pp. 831846 , Oct 2014

[21] S. Hur, T. Kim, D. Love, J. Krogmeier, T. Thomas, and A. Ghosh, "Millimeter wave beamforming for wireless backhaul and access in small cell networks," Communications, IEEE Transactions on, vol. 61, no. 10 , pp. 4391-4403, October 2013.

[22] J. Wang, "Beam codebook based beamforming protocol for multi-gbps millimeter-wave wpan systems," IEEE Journal on Selected Areas in Communications, vol. 27, no. 8, pp. 1390-1399, October 2009.

[23] T. S. Rappaport, F. Gutierrez, E. Ben-Dor, J. N. Murdock, Y. Qiao, and J. I. Tamir, "Broadband millimeter-wave propagation measurements and models using adaptive-beam antennas for outdoor urban cellular communications," IEEE Transactions on Antennas and Propagation, vol. 61, no. 4, pp. 1850-1859, April 2013.

[24] T. Rappaport, S. Sun, R. Mayzus, H. Zhao, Y. Azar, K. Wang, G. Wong, J. Schulz, M. Samimi, and F. Gutierrez, "Millimeter wave mobile communications for $5 \mathrm{~g}$ cellular: It will work!" Access, IEEE, vol. 1 pp. 335-349, 2013.

[25] M. Akdeniz, Y. Liu, M. Samimi, S. Sun, S. Rangan, T. Rappaport, and E. Erkip, "Millimeter wave channel modeling and cellular capacity evaluation," Selected Areas in Communications, IEEE Journal on, vol. 32, no. 6, pp. 1164-1179, June 2014

[26] A. Maltsev, R. Maslennikov, A. Sevastyanov, A. Lomayev, and A. Khoryaev, "Statistical channel model for $60 \mathrm{ghz}$ wlan systems in conference room environment," in Proceedings of the Fourth European Conference on Antennas and Propagation, April 2010, pp. 1-5. 